\title{
Enabling Technologies for a Practical Wireless Communication System Operating in TV White Space
}

\author{
Chin-Sean Sum, Gabriel Porto Villardi, Zhou Lan, Chen Sun, Yohannes Alemseged, \\ Ha Nguyen Tran, Junyi Wang, and Hiroshi Harada \\ Smart Wireless Laboratory, National Institute of Information and Communications Technology (NICT), Yokosuka, Japan \\ Correspondence should be addressed to Chin-Sean Sum, sum@nict.go.jp
}

Received 22 February 2011; Accepted 20 April 2011

Academic Editors: F. Benedetto and Y. Jiang

Copyright (c) 2011 Chin-Sean Sum et al. This is an open access article distributed under the Creative Commons Attribution License, which permits unrestricted use, distribution, and reproduction in any medium, provided the original work is properly cited.

This paper proposes the design of enabling technologies for practical wireless communication systems operating in the TV white space (TVWS). The main objective of this paper is to cover a macro perspective on the system design blocks including: (a) targeted use case applications and governing regulations, (b) channelization, physical (PHY) layer and medium access control (MAC) layer designs, and (c) achievable throughput and range. It is the intention of this paper to serve as a general guideline for designing wireless communication systems operating in TVWS. The core system design addresses both PHY and MAC layer issues with realistic system considerations. In the PHY layer, a channelization design that fits into the area-specific TV channels and a transceiver that enables data exchange in the TV bands are designed. In the MAC layer, a cognitive engine that manages access to vacant TV channels and MAC functionalities that facilitate effective medium access are also proposed. As a result, the system is capable of supporting up to a typical throughput of $80 \mathrm{Mbps}$, and a maximum number of 40 users, assuming all users performing the most bandwidth-hungry application in the use case scenario. The corresponding operating range is found reach up to $400 \mathrm{~m}$.

\section{Introduction}

The recent regulatory development [1-3] has opened up new opportunities for unlicensed wireless communication systems to utilize unoccupied TV channels (a.k.a TV White Space or TVWS) in the very high frequency (VHF) and ultrahigh-frequency (UHF) bands. TVWS is defined as the TV bands that can be occupied by secondary (often unlicensed) wireless systems with the condition that the related regulatory and technical requirements are met. The opening of TVWS has catalyzed more innovative and diversified wireless applications, and has invited overwhelming responses from the industrial community. Among others is the mobilization in international standardization initiatives [4-7].

It is, therefore, a timely window to provide to the technical community the enabling wireless technology for operation in the TVWS. The two core factors that drive the direction of the technology are: (a) potential application use cases that define and verify the necessity, and (b) governing regulations that dictate the "do's and do not's." The enabling technology shall consist of a system design capable of delivering the characteristics and performance as required by the targeted application use cases, and at the same time, capable of complying to the rules set by the regulations. For the design of TVWS enabling technology, it is of no exception. In order to validate its existence, the system has to possess a "killer-application". And in order to harvest and utilize the new spectrum resources, the system has to possess the appropriate enabling functionalities to operate in the vacant TV channels within the boundaries set by local primary-user protection regulations.

For this purpose, in this paper, we have proposed a full system design taking into consideration all three elements: the potential application use cases, the governing regulations, and the corresponding cross-layer system design. Firstly, the use cases highlight the prospective applications that outline several requirements in communication speed and range. Secondly, the governing regulations set conditions and boundaries that fractionally limits the liberty of the system to access the TVWS. Thirdly, our system design is 
specified to meet the use case requirements and to be able to comply to the regulations. The proposed cross-layer design is further divided into channelization design, physical (PHY) layer design, and cognitive medium access control (MAC) layer design. It should be emphasized that before detailed investigations and optimizations are conducted in each of these elements, it is essentially important to have the overall assessment that proves the feasibility of the technology in delivering the mandated task and to provide a high-level guidance in respective lower-level system design. To the best knowledge of the authors, such works are nonexistent in the current literature.

The contribution of this paper is threefold: (a) it verified that wireless technology operating in the TVWS does offer encouraging opportunities, (b) providing an overall crosslayer MAC and PHY cognitive system design that considers practical applications and regulations, and (c) providing realistic performance evaluation (e.g., throughput, coverage, etc.) in response to related requirements. It is our strong belief that this paper is of interest to both the academic and industrial community. The detailed design philosophy and step-by-step design procedure are given in Section 2. The combined discussion on how the requirements, design considerations, and system performance are related to each other is given in Section 6.

\section{General Design Philosophy}

This section gives an overview on the design philosophy and design flow of the TVWS enabling technology, as illustrated by Figure 1. The starting point of the TVWS often lies in the regulations and the use cases. In the case of TVWS, the development of regulations creates opportunities and requirements for the corresponding wireless technology. The opportunities have motivated the extended use cases of the technology, while the requirements affect the design of the system. Together, the regulations and the use case become the input factors that influence the system design. Sections 3 and 4 address these arenas.

In this paper, the system design consists of the channelization, the PHY layer, and the MAC layer design. The channelization design takes into consideration the specific TV channels allowed by different regulatory domains. In other words, the channelization engine has to be capable of supporting all the operable TV channels. In this work, a dual phase-locked loop (PLL) design is employed to produce both center frequencies and channel spacing. Section 5.1 provides a generic design with specific numerical examples.

The PHY layer intakes the output of the channelization design as input design parameters. The PHY design considers the transceiver design, the TV channel model, and the operating range. Among others, the operating range has to support the practical scenarios in the use cases. The output parameters of the PHY design such as error performance and PHY layer data rate will be sent to the MAC layer. Section 5.2 presents the practical PHY layer design for the communication system operating in the TVWS.

The MAC layer consists of the cognitive management engine and other MAC enabling protocols. The cognitive management engine deals with the occupancy of TVWS taking into consideration the required regulations. Section 5.3 describes the cognitive engine design. Besides the TVWS occupancy management engine, other MAC protocols are also essential to enable communication control and data communication. The output parameters of the MAC design such as the system throughput are crucial and will be the core reference to support the targeted use cases. Section 5.4 provides the MAC layer design with practical numerical values.

In a nutshell, this paper provides a practical cross-layer design for a practical communication system operating in the TVWS based on realistic numerical values in the real-world industrial design.

\section{Use Cases}

The typical use case of communication in TVWS is often associated with wireless coverage of wide areas (e.g., suburban and rural areas) where installation of facilities can be costly. In this perspective, the TVWS communication is especially a suitable candidate given its long reaching and high penetrating VHF/UHF signal characteristics. Figure 2 illustrates the typical scenario of a wide area high-speed backbone connectivity fed by respective local subnetworks. Sites A, B, and C are far-apart locations each with high-power devices (HPD) antenna towers linked via TVWS connection, while the low-power devices (LPD) may be portable/mobile devices linked to HPDs via TVWS connection. The clear definitions of HPD and LPD are outlined in accordance to regulations. This general illustration can be applied to, but not limited to the following.

(i) Campus. HPD—rooftop antenna of buildings. LPD—computers and mobile devices.

(ii) Industrial Site. HPD-antenna in control center. LPD—machineries and automation.

(iii) Municipality. HPD—municipal building antenna. LPD—employee workstations.

(iv) Enterprise. HPD—enterprise building antenna. LPD—employee workstations.

(v) Utility Network. HPD—antenna of utility provider. LPD—data collectors and wireless meters.

The above use case scenarios have been constantly addressed in various initiatives [8-11] calling for enablement of communication in the TVWS. It is, therefore, a practical "killer-application" based on popular demand.

In these typical scenarios, the parameter requirements are given in Table 1. For backbone connectivity between HPDs in different locations (e.g., building rooftops) several hundreds of meters apart, the required throughput is typically 50 to $80 \mathrm{Mbps}$. These HPD will then be connected to LPDs (e.g., access points) that distributes the bandwidth to respective end users. Depending on the applications, each end user may require throughput of $100 \mathrm{kbps}$ to $2 \mathrm{Mbps}$. For the $100 \mathrm{kbps}$ requirement, hundreds of end users can be supported. As for the $2 \mathrm{Mbps}$ requirement, tens of end users can be supported. 


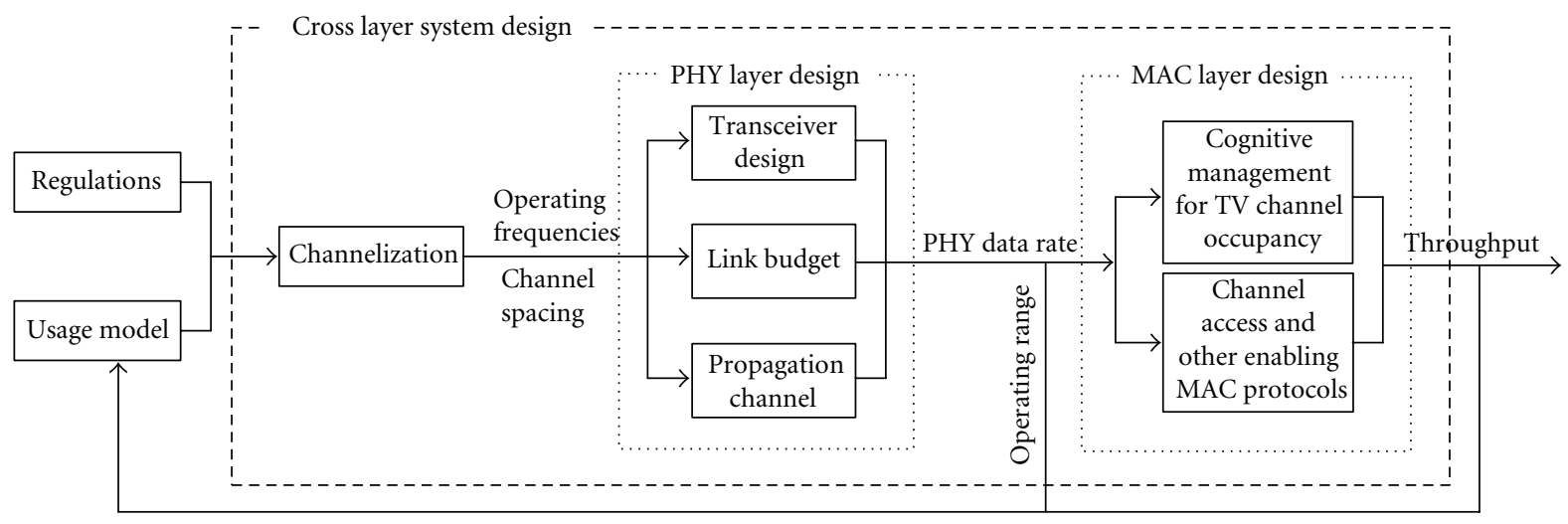

Figure 1: Cross-layer design philosophy and basic flow.

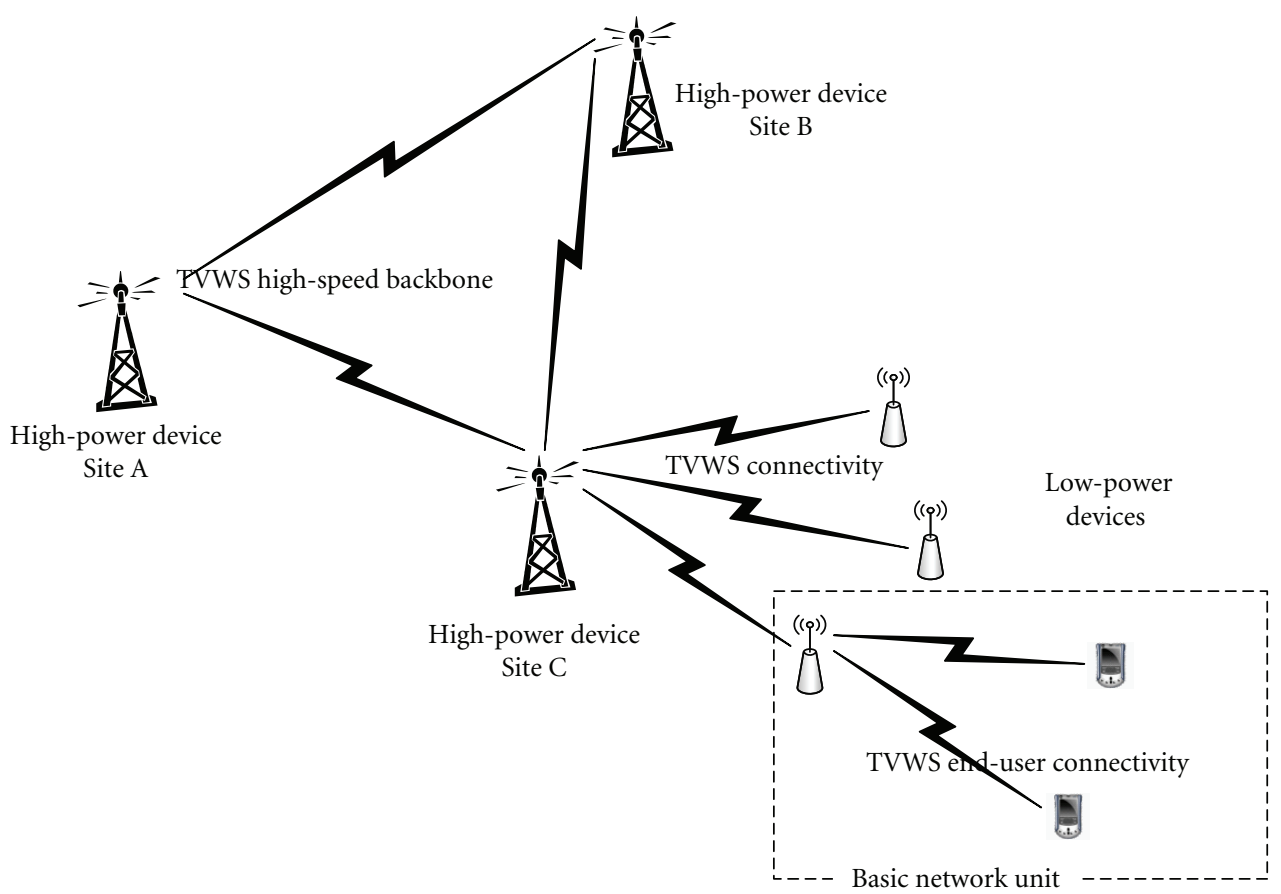

Figure 2: Typical use case example for TVWS communications.

TABle 1: Parameters Requirement for TVWS Use Cases.

\begin{tabular}{|c|c|c|c|}
\hline Parameter & & Requirement & \\
\hline Environment & & LOS and NLOS & \\
\hline Data rate class & Low rate & Medium rate & High rate \\
\hline Data rate & Up to $100 \mathrm{kbps}$ & Up to $2 \mathrm{Mbps}$ & Minimum 60 Mbps \\
\hline Average range & \multicolumn{2}{|c|}{ Several tens of meters } & Several hundreds of meters \\
\hline Connectivity type & \multicolumn{2}{|c|}{ End-user connectivity } & Backbone connectivity \\
\hline Use case examples & Wireless meters, sensor networks & Video streaming, web browsing & Backbone \\
\hline Required FER & \multicolumn{3}{|c|}{0.08} \\
\hline Number of end users & \multicolumn{2}{|c|}{ Several tens } & Several \\
\hline
\end{tabular}


From the perspective of applications, the $100 \mathrm{kbps}$ requirement mainly targets on sensor networks such as the smart utility networks [12], where low-power wireless meters are installed connected to wireless data collector. On the other hand, the $2 \mathrm{Mbps}$ requirement mainly targets on broadband access including online video streaming, web browsing, and local file transferring. Among these applications, the online video streaming (e.g., MPEG4 format [13]) requires a typical throughput of $2 \mathrm{Mbps}$. The end users are connected to the backbone that supports a typical speed of several tens (e.g., $60 \mathrm{Mbps}$ as given in Table 1) of Mbps.

\section{TVWS Regulations}

The occupancy of TVWS is closely tied to the policy and rulemaking of the regulatory institutions. Up-to-date, the several regulators actively participating in TVWS regulations are the United States (US) Federal Communications Commission (FCC), the United Kingdom (UK) Office of Communications (OFCOM), the European Conference of Postal and Telecommunications Administrations (CEPT), and the Japanese Ministry of Internal Affairs and Communications (MIC). Some of these regulations have already reached a fairly matured specification sufficient for actual system deployment, while others are still in the stage of planning and brain-storming. This paper places weighted emphasis on the more complete and matured FCC regulations.

FCC has issued a report and order (R\&O) [1] in Nov. 2008, and then later in Sept. 2010 [2], outlining the governing regulations for unlicensed usage in the TVWS. The TV band devices (TVBD) are categorized into two classes: fixed and personal/portable devices (hereon referred to as portable devices). The fixed device is a HPD operating at a fixed location with high-power outdoor antenna. The portable device is an LPD, and is further divided into Mode I and Mode II. A Mode I device is a client portable device controlled by a fixed device or a Mode II portable device. A Mode II device is an independent portable device with capability of accessing available TV channels.

Two cognitive radio capabilities are specified by the FCC for the TVBDs: the mandatory channel availability/geolocation awareness and the optional spectrum sensing. All devices, except Mode I portable devices, must include a location awareness capability by means of accessing the internet to obtain from a Geolocation Database (DB) information consisting of colocating incumbent TV broadcasting. Prior to operating, the unlicensed devices must access the geolocation DB to get the list of permitted channels currently not occupied by the incumbent services. Additionally, all devices may sense the spectrum for the presence of TV signals and wireless microphone signals. The sensitivity of the sensing is all the way down to a received power level of $-114 \mathrm{dBm}$.

In the US, the TV channels usable by unlicensed users range from the VHF channels 2-13 to the UHF channels 1451, while several limitations and exceptions apply [1]. Each TV channel is $6 \mathrm{MHz}$ wide. Table 2 shows a fraction of the UHF TV channels in the US.
TABLE 2: TV channels in the United Stated: an example of available TVWS. $f_{L}$ : lower frequency. $f_{H}$ : upper frequency.

\begin{tabular}{lccc}
\hline $\begin{array}{l}\text { TV channel } \\
\text { no. }\end{array}$ & $\begin{array}{c}\text { Frequency band } \\
f_{L}-f_{H}(\mathrm{MHz})\end{array}$ & $\begin{array}{c}\text { TV channel } \\
\text { no. }\end{array}$ & $\begin{array}{c}\text { Frequency band } \\
f_{L}-f_{H}(\mathrm{MHz})\end{array}$ \\
\hline 38 & $614-620$ & 45 & $656-662$ \\
39 & $620-626$ & 46 & $662-668$ \\
40 & $626-632$ & 47 & $668-674$ \\
41 & $632-638$ & 48 & $674-680$ \\
42 & $638-644$ & 49 & $680-686$ \\
43 & $644-650$ & 50 & $686-692$ \\
44 & $650-656$ & 51 & $692-698$ \\
\hline
\end{tabular}

It should be noted that FCC is only one among the many regulatory organizations that outlined governing rules for operation in the TVWS. There are many other regulators actively working on similar rules and regulations. In this paper, although uses the examples and specific parameters of FCC, the same analysis can be repeated for other regulations.

\section{System Design for Enabling TVWS Operation}

In this section, system requirements inherent to the use cases as described in Section 3, and regulatory demands as described in Section 4, are taken into consideration for the overall system design. The cross-layer system design is divided into channelization, PHY layer design, cognitive management, and MAC layer design.

5.1. Channelization. The first essential parameter in enabling communication in TVWS is the channelization strategy. The communication system has to be equipped with the capability to operate at center frequencies and channel spacing of unused TV channels. For this purpose, a clock generator consisting of crystal oscillator and phase-locked loop (PLL) is designed. The clock generator employs a dualPLL structure to produce both the center frequencies and symbol rate (i.e., Nyquist frequency) $f_{\text {nr }}$.

Figure 3 shows the architecture of the clock generator. In Figure 3-center frequency generation, the phase detector compares the two input signals $f_{\mathrm{ic}}$ and $f_{\mathrm{bc}}$ to obtain the error signal that is proportional to their phase difference. The error signal is then low-pass filtered (LPF) and used to drive a voltage-controlled oscillator (VCO) which creates an output frequency, $f_{\text {cen }}$. The output frequency is fed through a frequency divider back to the input system as $f_{\mathrm{bc}}$, thus producing a feedback loop. If $f_{\text {cen }}$ drifts, the error signal will increase, driving the VCO frequency in the opposite direction so as to reduce the error. Therefore, the output is locked to the input frequency $f_{\text {ic, }}$, which is the product of a stable frequency $f_{\text {XTAL }}$ derived from a crystal oscillator. This is the basic design to produce the center frequency of the TV channels. In Figure 3-symbol rate generation, the symbol rate $f_{\text {nr }}$ can also be obtained by using the same procedure, with a different set of parameters.

By employing the designed dual-PLL clock, the specific center frequencies $f_{\text {cen }}$ of the US TV channels given in Table 2 


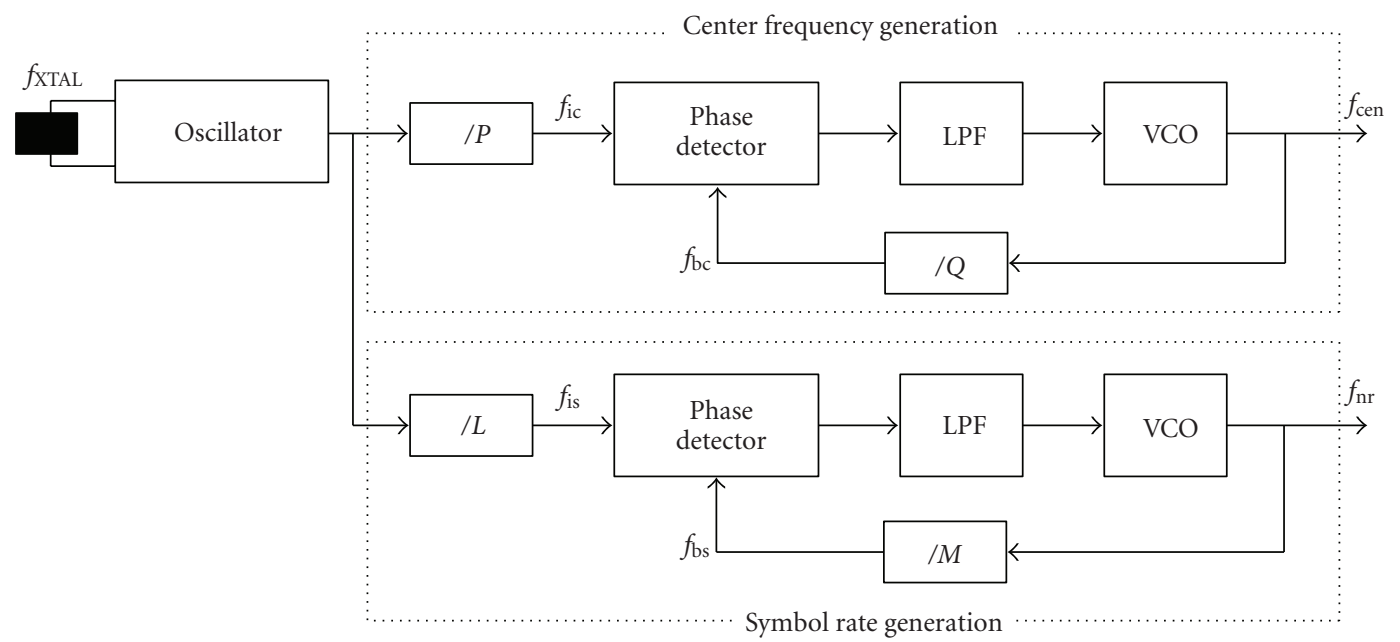

FIGURE 3: Dual-PLL reference diagram for clock generator.

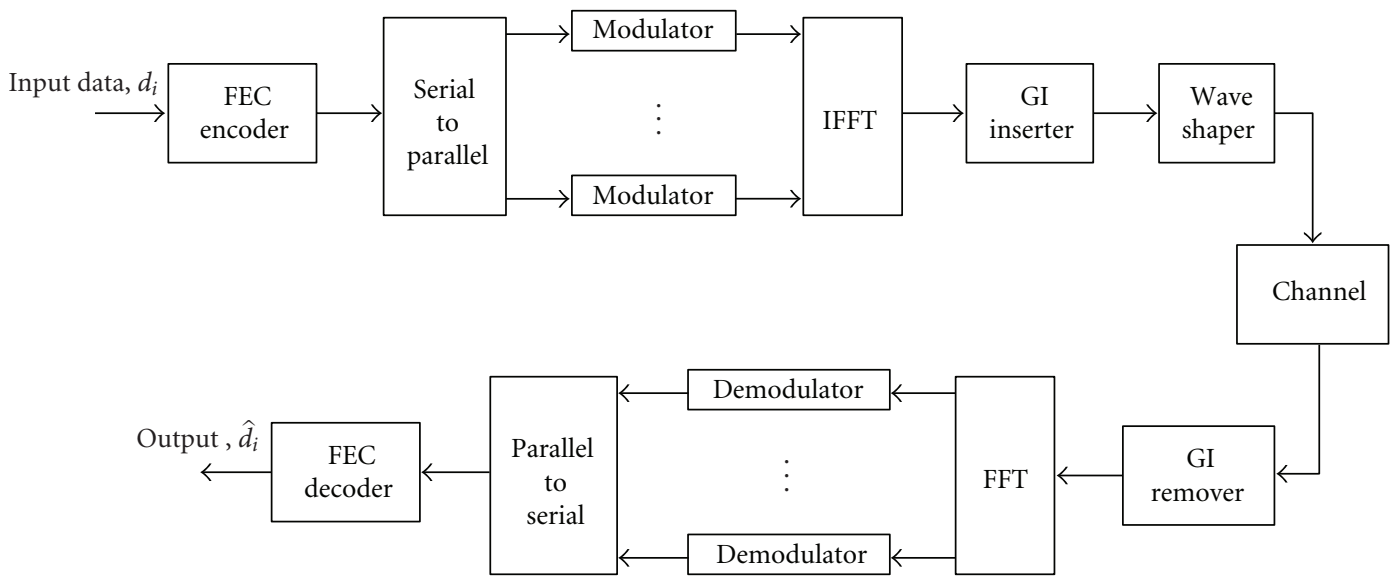

FIgURE 4: Block diagram for OFDM transceiver.

can be generated. In this numerical example, the $40 \mathrm{MHz}$ crystal oscillator (i.e., $f_{\mathrm{XTAL}}=40 \mathrm{MHz}$ ), a popular choice for most 802.11 wireless local area network (WLAN) systems [14] is assumed. In Figure 3, by substituting $P=f_{\text {XTAL }}, f_{\text {ic }}$ of $1 \mathrm{MHz}$ can be obtained. Then, by employing $Q=f_{L}+\left(f_{H}-\right.$ $\left.f_{L}\right) / 2$ where $f_{H}$ and $f_{L}$ are upper and lower frequencies of the TV channels as shown in Table 2, $f_{\text {cen }}$ for each TV channel can be generated. Similarly, by substituting $L=1, f_{\text {is }}=f_{\mathrm{XTAL}}$ can be produced. Next, by using $M=8,4$, and 2 , symbol rate $f_{\mathrm{nr}}$ of 5,10 , and $20 \mathrm{MHz}$ can be generated. Note that the reason to select symbol rates of 5,10 and $20 \mathrm{MHz}$ is also motivated by the attempt to align the TVWS communication system to the popular WLAN.

While the specific numerical results are derived assuming particular regulations and existing wireless systems, the generic clock architecture is capable of supporting frequency generation in any band and channel spacing.

5.2. PHY Layer Design. The channel plan provided by the generator in Section 5.1 gives the starting point of designing the PHY layer. In the following sections, the PHY layer design will be described, with the achievable PHY data rate and operating range as the output parameter.

5.2.1. Signal Model. Figure 4 shows an illustration of the orthogonal frequency division multiplexing (OFDM) transmission system for the TVWS operation. In the transmitter, the high-speed data is first encoded with a forward error correction (FEC) scheme with coding rate $r_{c}$, and then converted into parallel data of subchannels. The transmitted data can be expressed as [15]

$$
\begin{gathered}
s(t)=\sum_{k=-\infty}^{\infty} \sum_{i=0}^{L_{\mathrm{FFT}}-1} d_{i}(k) \exp \left(j 2 \pi f_{i}\left(t-k T_{\text {sym }}\right)\right) \\
\times f_{\text {cen }}\left(t-k T_{\text {sym }}\right)
\end{gathered}
$$

where $L_{\mathrm{FFT}}$ is the total number of OFDM subcarrier, $T_{\text {sym }}$ is the OFDM symbol duration, $f_{i}$ is the frequency of the $i$ th subcarrier, and $f_{i}=f_{\text {cen }}+i / T_{\text {sym }}, f_{\text {cen }}$ is the operating frequency. Note that each data $d_{i}$ is a complex number, $d_{i}=d_{I_{i}}+j d_{Q_{i}}$, where $d_{I_{i}}$ and $d_{Q_{i}}$ are in the range of 
$\{ \pm 1,0\},\{ \pm 1, \pm 1\},\{ \pm 3, \pm 3\}$ and $\{ \pm 7, \pm 7\}$ for binary phase shift keying (BPSK), quadrature phase shift keying (QPSK), 16 quadrature amplitude modulation (16QAM), and 64 quadrature amplitude modulation (64QAM), respectively. The modulation level $m_{l}$ for these schemes are 1, 2, 4, and 6 , respectively. The chosen FEC scheme is the Reed-Solomon block codes.

Here, the subcarrier spacing $\zeta_{\text {FFT }}$ of the OFDM signal and its equivalent time domain representation $T_{\mathrm{FFT}}$ are given as

$$
\zeta_{\mathrm{FFT}}=\frac{f_{\mathrm{nr}}}{L_{\mathrm{FFT}}}, \quad T_{\mathrm{FFT}}=\frac{1}{\zeta_{\mathrm{FFT}}},
$$

where $f_{\mathrm{nr}}$ is the Nyquist frequency produced by the clock generator.

After the insertion of guard interval (GI), the OFDM signal is described as

$$
s(t)^{\prime}=\sum_{k=-\infty}^{\infty} \sum_{i=0}^{L_{\mathrm{FFT}}-1} d_{i}(k) \exp \left(j 2 \pi f_{i}\left(t-k T_{\text {total }}\right)\right) f^{\prime}\left(t-k T_{\text {total }}\right),
$$

where $T_{\text {total }}=T_{\text {sym }}+T_{\mathrm{GI}}, T_{\mathrm{GI}}$ is the GI interval, $f^{\prime}(t)$ is the modified pulse waveform of each symbol defined as $f^{\prime}(t)=1$ if $-T_{\mathrm{GI}} \leq t \leq T_{\mathrm{sym}}$, and $f^{\prime}(t)=0$ if $t<-T_{\mathrm{GI}}$ and $t>T_{\mathrm{sym}}$.

5.2.2. Receiver Model. At the receiver, the received signal is given by

$$
r(t)=\int_{0}^{\infty} h(\tau, t) s(t-\tau) d \tau+\eta(t)
$$

where $h(\tau, t)$ is the impulse response of the radio channel at time $t$, and $\eta(t)$ is the complex additive white Gaussian noise (AWGN). The receiver configuration is described in Figure 4. The output of the receiver is expressed as the $i$ th OFDM subcarrier as

$$
\hat{d}_{i}=\frac{1}{T_{\text {sym }}} \int_{k T_{\text {total }}}^{T_{\text {sym }}+k T_{\text {total }}} r(t) \exp \left(-j 2 \pi f_{i}\right)\left(t-k T_{\text {total }}\right) \mathrm{d} t
$$

Note that $\hat{d}_{i}$ will be used to compare with the input data to obtain the error performance.

5.2.3. Design Parameter Considerations. This subsection presents the essential PHY parameters in designing the OFDM signaling. The output of the PHY layer design are the PHY data rate $\Omega_{\mathrm{PHY}}$ and the operating range $\Upsilon$, that shall be sufficient to support the requirements in the targeted use cases, as given in Table 1. In order to achieve the sufficient $\Omega_{\text {PHY }}$ and $\Upsilon$, the critical parameters to be considered are modulation level $m_{l}$, FEC coding rate $r_{c}$, FFT size $L_{\mathrm{FFT}}$, and GI size $L_{\mathrm{GI}}$.

In the VHF/UHF band, existing channel propagation studies that are widely used are reported in $[16,17]$. The important parameters extracted from these studies are the maximum delay spread $\epsilon_{\max }$ that dictates the design of the OFDM signaling. In [16], $\epsilon_{\max }$ is given as $10-20 \mu$ s for the range of $10-20 \mathrm{~km}$. In [17], $\epsilon_{\max }$ is $11-25 \mu \mathrm{s}$ for $17-33 \mathrm{~km}$ and $25-60 \mu \mathrm{s}$ for up to $100 \mathrm{~km}$. As a result, it is concluded that within $10 \mathrm{~km}, \epsilon_{\max }$ can be set to $12 \mu \mathrm{s}$. Next, in order to effectively mitigate frequency selective fading in the OFDM signal, $T_{\mathrm{GI}}$ should be set larger than $\epsilon_{\max }$.

For backbone connectivity, $L_{\mathrm{FFT}}$ is chosen to be 1024 for the HPD, while for end-user connectivity, $L_{\mathrm{FFT}}$ is chosen to be 256 for the LPD for lower complexity. For both scenarios, $T_{\mathrm{GI}}$ is set to $T_{\mathrm{FFT}} / 4$. The Nyquist frequency $f_{\mathrm{nr}}$ for backbone connectivity and end-user connectivity are set to 5 and $20 \mathrm{MHz}$, respectively. These settings provide to both the backbone connectivity and end-user connectivity, $T_{\mathrm{GI}}>$ $\epsilon_{\max }=12.8 \mu \mathrm{s}$. In other words, the frequency selective fading can be mitigated with these settings.

5.2.4. Error Performance. The bit error rate (BER) can be quantified by comparing the transmitted bits $d_{i}$ and the bits produced by the receiver $\hat{d}_{i}$ in (5), as

$$
\begin{aligned}
\operatorname{BER}= & P\left(d_{i}=+1\right) P\left(\hat{d}_{i}=-1 \mid d_{i}=+1\right) \\
& +P\left(d_{i}=-1\right) P\left(\hat{d}_{i}=+1 \mid d_{i}=-1\right),
\end{aligned}
$$

where $P(\cdot)$ denotes the probability and $d_{i}$ and $\hat{d}_{i}$ represent the $i$ th transmitted and received data bit.

In similar way, the frame error rate (FER) can be described as

$$
\begin{array}{r}
\text { FER }=P\left(d_{j}=\varrho_{j}\right) P\left(\widehat{d_{j}} \neq \varrho_{j} \mid d_{j}=\varrho_{j}\right), \\
j=1,2, \ldots, L_{\mathrm{fr}},
\end{array}
$$

where $d_{j}$ and $\widehat{d}_{j}$ represent the $j$ th transmitted and received data bit in a data frame, $\varrho_{j}$ is the $j$ th bit from the data bit sequence of length $L_{\mathrm{fr}}$, sent as a transmitted frame. The relationship between FER and BER can be expressed by

$$
\operatorname{FER}=1-\left((1-\mathrm{BER})^{L_{\mathrm{fr}}}\right)
$$

Specific numerical values are substituted into the PHY layer design to determine the error performance of the system. For this purpose, the FEC Reed-Solomon coding is designed to encode 239 to 255 block symbols. The chosen modulation schemes are QPSK, 16QAM, and 64QAM. The propagation channel is assumed to be a fading channel with Rayleigh distribution to consider the bottleneck of the channel condition. The signal to noise ratio (SNR) in the receiver is quantified as the ratio between energy per bit $\left(E_{b}\right)$ to noise power spectral density $\left(N_{0}\right)$. In this paper, we neglect the intercarrier-interference between OFDM subcarriers for simplicity.

Figure 5 shows the error performance of the OFDM system in a fading channel with Rayleigh distribution. The minimum performance requirement for the system is application-dependent, as given in Table 1. By using (8), the required BER can be approximated as $10^{-5}$ with typical $L_{\mathrm{fr}}=4096$ [14]. Figure 5 gives the equivalent required $E_{b} / N_{0}$ for QPSK, 16QAM, and 64QAM to be 23, 25.5, and $29 \mathrm{~dB}$, respectively. 
5.2.5. PHY Data Rates. This section calculates the achievable PHY data rate $\Omega_{\mathrm{PHY}}$ based on the design parameters discussed in Section 5.2.3. The relationship between $\Omega_{\mathrm{PHY}}$ and relevant design parameters can be expressed as

$$
\Omega_{\mathrm{PHY}}=f_{\mathrm{nr}} m_{l} r_{c} \frac{L_{\mathrm{FFT}}-L_{\mathrm{GI}}}{L_{\mathrm{FFT}}} .
$$

Employing (9), the list of supported $\Omega_{\mathrm{PHY}}$ are given in Table 3. In Table 3, Index 1 to 3 are designed for end-user connectivity which requires up to a total of $2 \mathrm{Mbps}$ per user according as specified in Table 1. Index 4 to 6 are designed to fulfill the requirements of the backbone connectivity, suiting to various operating ranges $\Upsilon$. The analysis on $\Upsilon$ will be conducted in Section 5.2.6. The combined analysis incorporating $\Omega_{\mathrm{PHY}}, \Upsilon$ and the use case requirements will be conducted in Section 6 .

5.2.6. Link Budget. Employing the PHY parameter settings in Section 5.2.5, the operating range $\Upsilon$ of data communication can be determined as

$$
\log _{10}(\Upsilon)=\frac{\left(P_{\mathrm{TX}}+G_{\mathrm{TX}}+G_{\mathrm{RX}}-S_{m}-L_{\mathrm{PLn}}\right)}{10 \phi},
$$

where $P_{\mathrm{TX}}$ and $G_{\mathrm{TX}}$ are the transmitter power and antenna gain, $G_{\mathrm{RX}}$ is the receiver antenna gain, and $\phi$ is the propagation loss index. Here, $L_{\mathrm{PLn}}$ is the well-known urban path loss model [18] and is not repeated in this paper. Note that $L_{\text {PLn }}$ is a normalized parameter with center frequency $f_{\text {cen }}=$ $600 \mathrm{MHz}$ (a typical value assumed in this example), HPD and LPD antenna height of $30 \mathrm{~m}$ and $1 \mathrm{~m}$, respectively. All parameters are in $\mathrm{dB}$ scale. The minimum receiver sensitivity $S_{m}$ is calculated as

$$
S_{m}(\mathrm{~dB})=-174+10 \log _{10}\left(\Omega_{\mathrm{PHY}}\right)+\mathrm{NF}+\frac{E_{b}}{N_{0}},
$$

where NF is the receiver noise figure, and $E_{b} / N_{0}$ is the required SNR to reach the targeted BER.

By substituting the relevant PHY parameters into (10), the effective operating range $\Upsilon$ can be determined. In this analysis, $P_{\mathrm{TX}}$ is set to $100 \mathrm{~mW}$ for LPD and $4 \mathrm{~W}$ for HPD as specified by [1], $G_{\mathrm{TX}}$ and $G_{\mathrm{RX}}$ are set to $0 \mathrm{dBi}$ to indicate omnidirectional transmission, $\mathrm{NF}$ is set to $8 \mathrm{~dB}$, and $\phi$ is set to 3. Other relevant parameters are set similar to the previous sections.

The achievable $\Upsilon$ is given in Table 3 . In Table 3, Index 1 to 3 are targeted for end-user connectivity and is thus confined within a shorter range between end users to the access point. On the other hand, Index 4 to 6 are intended for backbone connectivity which requires higher effective operating range to bridge across fixed stations. The combined analysis incorporating $\Omega_{\mathrm{PHY}}, \Upsilon$ and the use case requirements will be conducted in Section 6 .

5.3. Cognitive Management Plane. The cognitive management plane of the TVWS communication system consists of a master protocol, namely, the Dynamic Enabling Protocol (DEP) that controls the access to the targeted TV channel. The cognitive management engine includes into the DEP mechanism the features of the channel availability/geolocation awareness and spectrum sensing. Figure 6 shows the typical state machine implementation of the DEP.

For fixed and portable Mode II devices, the channel availability/geolocation awareness through accessing the $\mathrm{DB}$ is a mandatory requirement while the spectrum sensing is an optional feature.

(i) Upon power on, a device is in the "unenabled" state. In the "unenabled" state, the device is not allowed to transmit any energy into the air. Next, the "unenabled" device accesses the DB containing information of incumbent users through the internet to inquire the vacancy of the targeted channel to be used. If the DB indicates that the targeted channel is not occupied by an incumbent user, the state transits to the "becoming enabled" state.

(ii) In the "becoming enabled" state, if spectrum sensing is activated, the device may still not transmit energy into the air. The device shall perform spectrum sensing to check the availability of the targeted channel. Upon determining the targeted channel to be vacant through spectrum sensing, the state of the device can then transit to the "enabled" state. This step is not necessary if spectrum sensing is activated. Additionally, at any time in the "becoming enabled" state, if a de-enablement signal is received from the $\mathrm{DB}$, indicating that an incumbent user has occupied the same channel, the device shall transit back to the "unenabled" state.

(iii) In the "enabled" state, the device may start to set up networks and perform data communication by transmitting signals into the air. At any time in the "enabled" state, the device transits to the "becoming enabled" state if a de-enablement signal is received from any other "enabled" device within the same channel OR if the spectrum sensing reports new activities of incumbent systems in the channel.

For portable Mode I devices, the state machine starts from the "becoming enabled" state. The rest of the state transition processes are the same.

5.4. MAC Layer Design. Following the DEP described in Section 5.3, a device in the "enabled" state is allowed to transmit signal into the air. This section further specifies the basic MAC layer design necessary for enabling communication in the TVWS.

5.4.1. Network Topology and Resource. A typical network consists of $N$ devices, one of which is selected as the network coordinator (NC). The remaining $N-1$ will be dependent devices associated with the NC. The NC provides the network reference timing and manages the resource sharing among the devices. A basic network unit is illustrated in the dotted box in Figure 2.

Figure 7 shows the reference timing for the network controlled by the NC. A full cycle of the network timing is 
TABLE 3: Achievable PHY data rate $\Omega_{\mathrm{PHY}}$ and achievable operating range $\Upsilon$.

\begin{tabular}{lcccccccccc}
\hline Index & $f_{\mathrm{nr}}(\mathrm{MHz})$ & Mod. & $m_{l}$ & $r_{c}$ & $L_{\mathrm{GI}}$ & $L_{\mathrm{FFT}}$ & $\Omega_{\mathrm{PHY}}(\mathrm{Mbps})$ & Dev. type & $P_{\mathrm{TX}}(\mathrm{mW})$ & $\Upsilon(\mathrm{m})$ \\
\hline 1 & 5 & QPSK & 2 & 0.9373 & 16 & 256 & 7 & LPD & 100 \\
2 & 5 & 16QAM & 4 & 0.9373 & 16 & 256 & 14 & LPD & 100 \\
3 & 5 & 64QAM & 6 & 0.9373 & 16 & 256 & 21 & LPD & 100 \\
4 & 20 & QPSK & 2 & 0.9373 & 256 & 1024 & 28 & HPD & 4000 & 210 \\
5 & 20 & 16QAM & 4 & 0.9373 & 256 & 1024 & 56 & HPD & 4000 \\
6 & 20 & 64QAM & 6 & 0.9373 & 256 & 1024 & 84 & HPD & 4000 & 440 \\
\hline
\end{tabular}

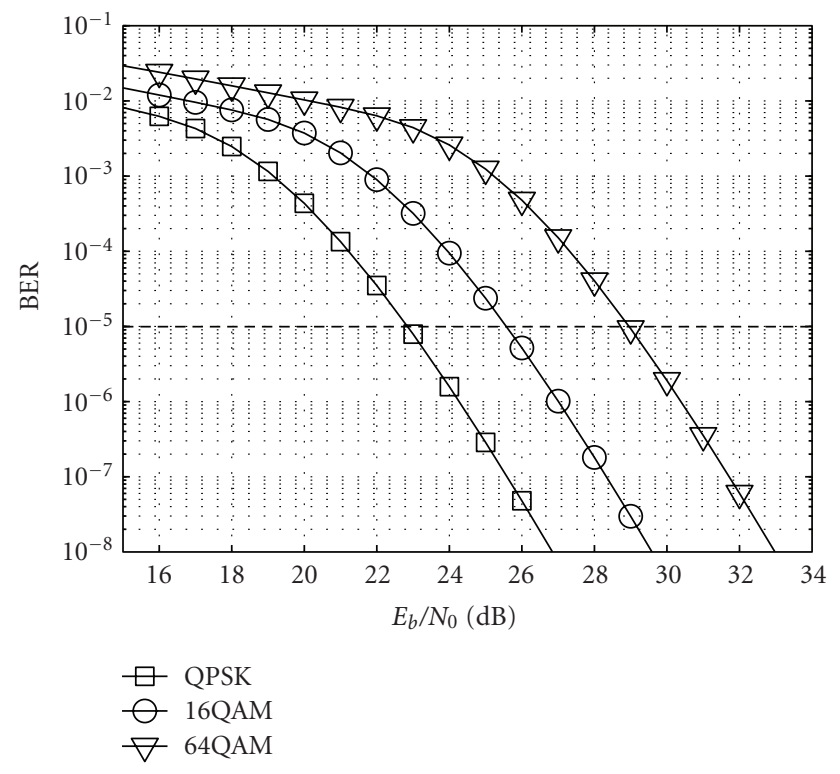

FIgURE 5: BER versus $E_{b} / N_{0}$ for fading channel with Rayleigh distribution. FEC: Reed-Solomon $(255,239)$.

known as the beacon interval (BI), which is the duration between two consecutive beacons. Following the beacon is a duration known as the contention access period (CAP). CAP is a duration that allows devices to access the wireless channel employing the carrier sense multiple access/collision avoidance (CSMA/CA) scheme. Following CAP is the contention free period (CFP). CFP allows the devices to access the wireless channel by using the time division multiple access (TDMA) scheme. In CFP, guaranteed time slots (GTSs) are allocated for each device to perform respective noncontentious communication.

The major reason of having both CAP and CFP for the MAC layer design is to support transmissions with and without stringent latency demands. As described in Section 3, the targeted use cases include local file transfer, web browsing, and video streaming. Particularly in video streaming, latency can be a delicate issue. Therefore, it is essential to have the hybrid CAP/CFP design to fulfill all needs.

5.4.2. MAC Functionalities. In order to enable communication in the TVWS, the MAC functionalities have to be specified. The basic set of functionalities to facilitate operation in the TVWS are starting a network, association/disassociation, GTS allocation, and data streaming, as shown in the message sequence charts (MSC) in Figure 8.

Starting a network involves the scanning process followed by the transmission of a beacon signal. In Figure $8(\mathrm{a})$, the MAC of a device receives request from the MAC Layer Management Entity (MLME) to scan all channels 1 to $M$. After the scan is completed, the MAC will report the results to the MLME. Next, a beacon frame will be sent by the device (now known as the NC) to seek other devices in the same location to form a network.

As shown in Figure 8(b), if a device receives a beacon frame and intends to join the network, the MLME will send an association request to the MAC. The MAC will then send an association request frame over the air to the NC. Upon receiving the request frame, the $\mathrm{NC}$ acknowledges with an acknowledgment (ACK) frame. The NC will then process the request and respond accordingly by sending the associate response frame. Similarly, the device acknowledges after receiving the associate response frame and reports to the MLME.

Figure $8(\mathrm{c})$ shows the GTS allocation for occupying the CFP. The device intending to occupy a GTS sends a GTS request frame to the NC. The NC acknowledges with an ACK frame, then sends to the MLME the request. In the next beacon transmission, the $\mathrm{NC}$ will insert into the beacon frame, the timing information of the GTS allocated for the device. The device upon receiving the beacon will wait until the CFP period and its turn to perform data streaming.

In Figure 8(d), data streaming is described. Assuming that the GTS notification is received by the preceding beacon, the device will send data frame to the destination (e.g., the NC) in the allocated GTS. The destination device will reply with an ACK frame if the data frame arrives.

5.4.3. Channel Access. As mentioned in Section 5.4.1, the two channel access methods to support data transmission in the TVWS are CSMA/CA in CAP and TDMA in CFP. This hybrid access method is a popular choice of major industrial standards specifications $[14,19,20]$. The following sections evaluates the achievable throughput for both schemes based on the data rate and other parameters provided by the PHY layer.

In the CSMA/CA scheme, a device attempting to transmit a frame first scan the TV channel. If the channel is idle for a period larger than $\alpha$, the device will transmit. If the device 


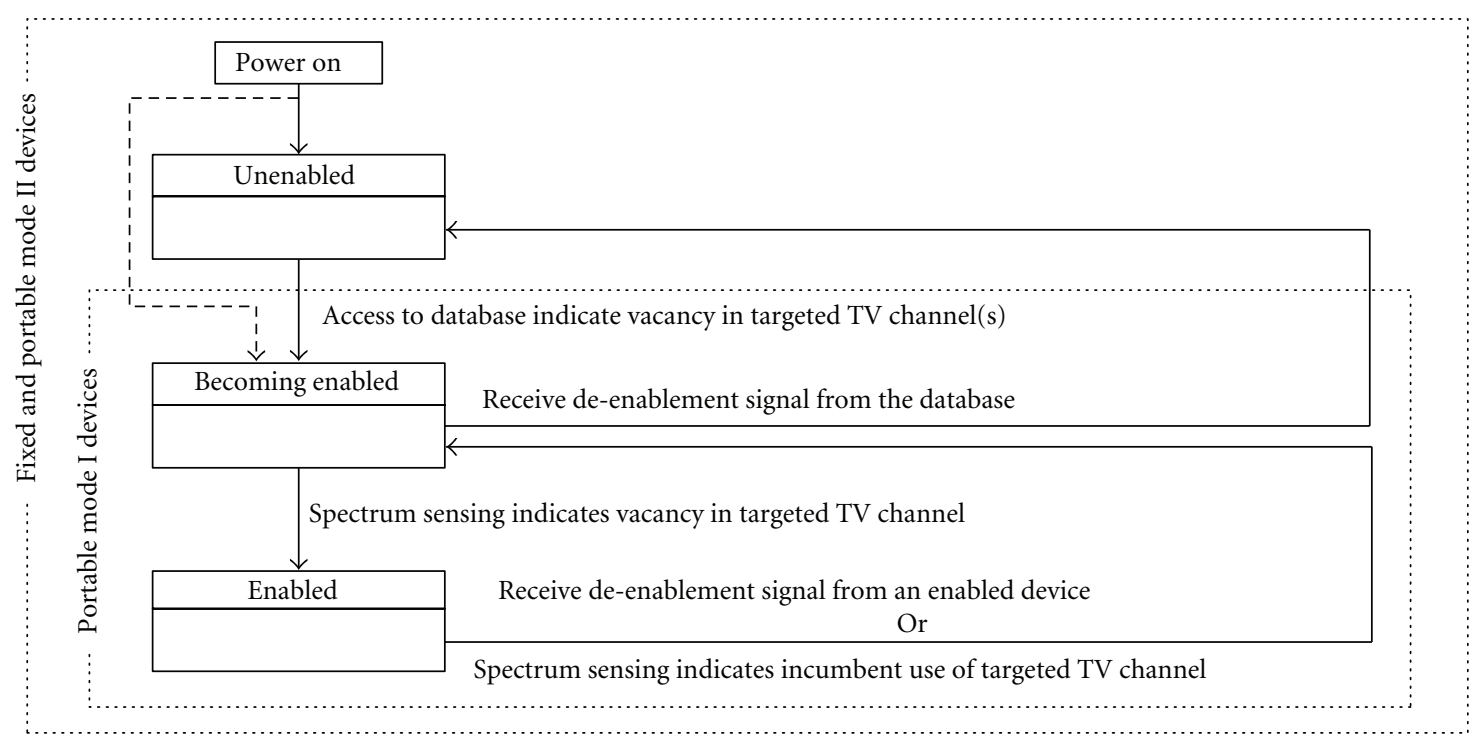

Figure 6: Cognitive management engine: Dynamic Enablement Protocol.

Time

\begin{tabular}{|l|c|c|c|c|c|c|c|c|}
\hline \multirow{3}{*}{ Beacon } & \multirow{2}{*}{ CAP } & \multicolumn{3}{|c|}{ CFP } & \multirow{3}{*}{ Beacon } & CAP & \multicolumn{3}{|c|}{ CFP } \\
\cline { 3 - 3 } & GTS1 & GTS2 & $\ldots$ & & GTS1 & GTS2 & $\ldots$ \\
\hline
\end{tabular}

FIGURE 7: Basic network reference timing of the NC.

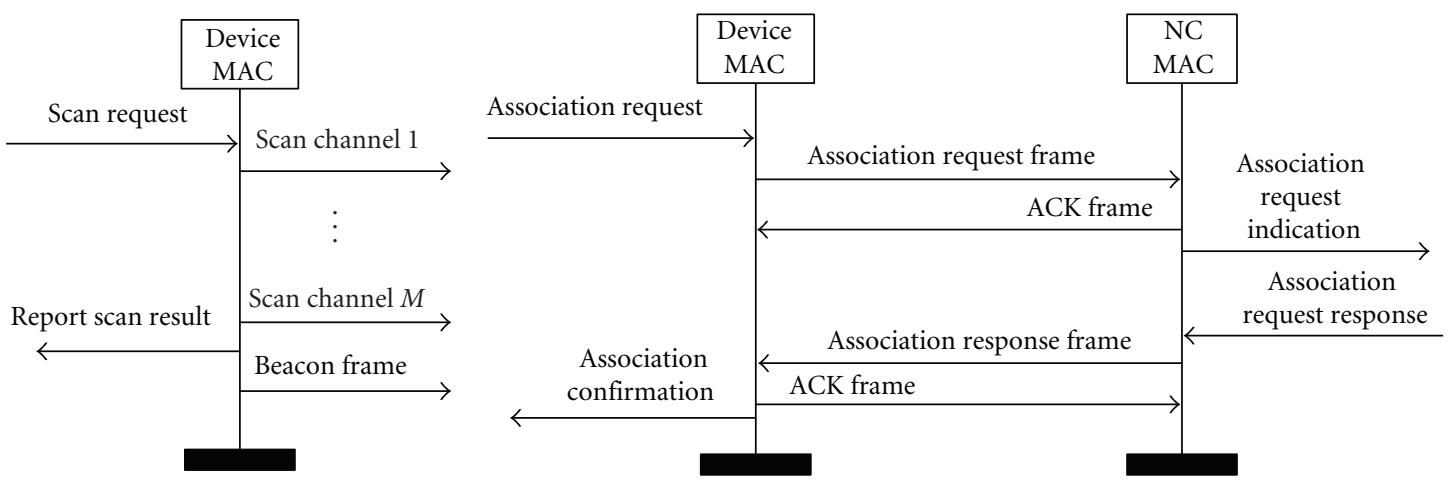

(a) Starting a network

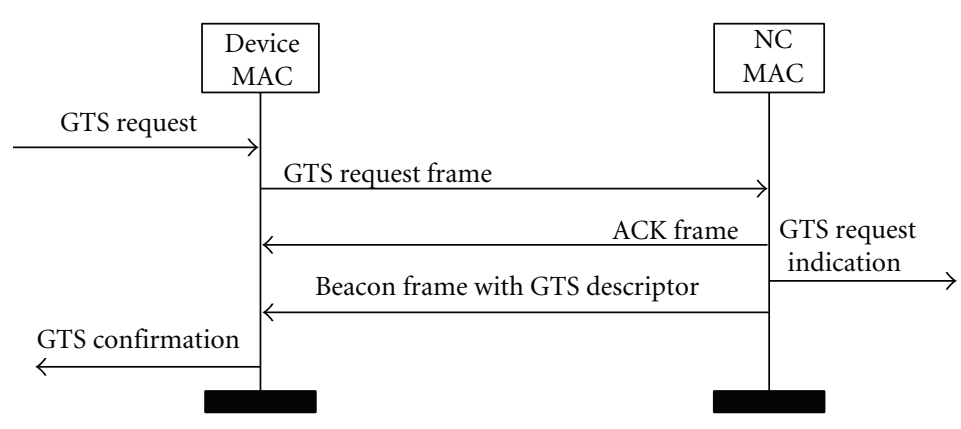

(c) GTS Allocation (b) Association

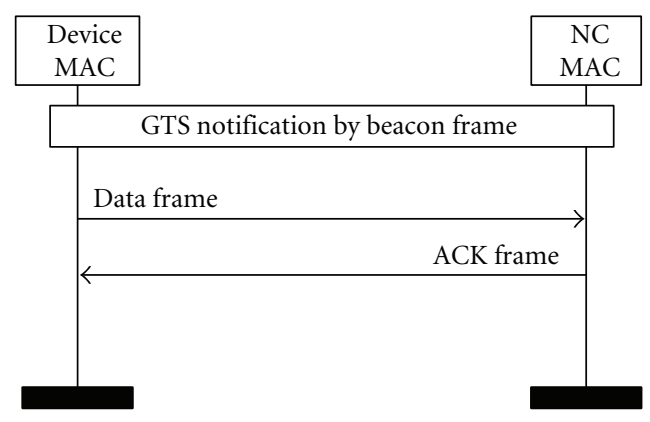

(d) Data streaming

FIGURE 8: MSC for basic MAC functions in TVWS communication system. 
detects that the channel is busy, it will persist to monitor the channel until it is idle. At this point, the device generates a random back-off interval to minimize collisions with frames from other devices. The back-off scale is discrete in time. The time following $\alpha$ is slotted, and a device is allowed to transmit only at the beginning of each slot time $\sigma$. Slot time $\sigma$ is a value dependent on the PHY layer design, taking into consideration the radio design such as propagation delay, transmitter-receiver-turn-around time, and so on.

The CSMA/CA uses an exponential back-off scheme. At each frame transmission, the back-off time is uniformly chosen in the range $(0, w-1)$, where $w$ is the contention window dependent of number of failed transmissions. At the first transmission attempt, $w$ is set to the minimum value $\mathrm{CW}_{\text {min }}$. Each unsuccessful transmission increases $w$ until the maximum value $\mathrm{CW}_{\max }$.

The transmitting device has no means of detecting a potential collision of the frames sent out, it is essential to also specify a feedback loop for the receiving device to acknowledge the successful receipt of frames. The ACK frame is transmitted by the destination device to the source device after receiving the whole frame. The interval between the end of the frame and the beginning of the ACK frame is noted as $\beta$. The system designed should be tailored to have $\beta<\alpha$, so that no other devices is able to detect the channel as idle during the ACK frame feedback.

In order to calculate the effective system throughput, we firstly express $\Lambda$ as the probability of a device transmitting in a randomly chosen slot time. Probability $\Lambda$ can be expressed as $[21,22]$

$$
\Lambda=\frac{2(1-2 p)}{(1-2 p)\left(\mathrm{CW}_{\min }+1\right)+p \cdot \mathrm{CW}_{\min }\left(1-(2 p)^{m}\right)},
$$

where $p$ is the probability that a transmitted frame encounters a collision and $m$ is the maximum back-off stage such that $\mathrm{CW}_{\max }=2^{m} \mathrm{CW}_{\min }$. In the simplified case of $m=0$ (i.e., no exponential backoff is considered), (12) can be rewritten as

$$
\Lambda^{(m=0)}=\frac{2}{\mathrm{CW}_{\min }+1} .
$$

Let $T_{I}$ be the idle duration. When all devices are counting down, no frame transmissions take place; thus,

$$
T_{I}=\sigma .
$$

Let $T_{\mathrm{SE}}$ be the success/error duration. When a frame is successfully transmitted or it is corrupted due to channel noise, the slot duration is given by

$$
T_{\mathrm{SE}}=T_{\mathrm{pre}}+T_{H}+T_{\mathrm{fr}}+\beta+\delta+\mathrm{ACK}+\alpha+\delta,
$$

where $T_{H}$ is the total header duration including the PHY and MAC headers, $T_{\text {pre }}$ is the duration of the preamble, $T_{\mathrm{fr}}$ is the payload duration, and $\delta$ is the propagation delay.

Let $T_{C}$ be the collision duration. When two or more devices transmit at the same time and collision occurs, the sender will wait for the next transmission. So,

$$
T_{C}=T_{\text {pre }}+T_{H}+T_{\text {fr }}+\alpha+\delta .
$$

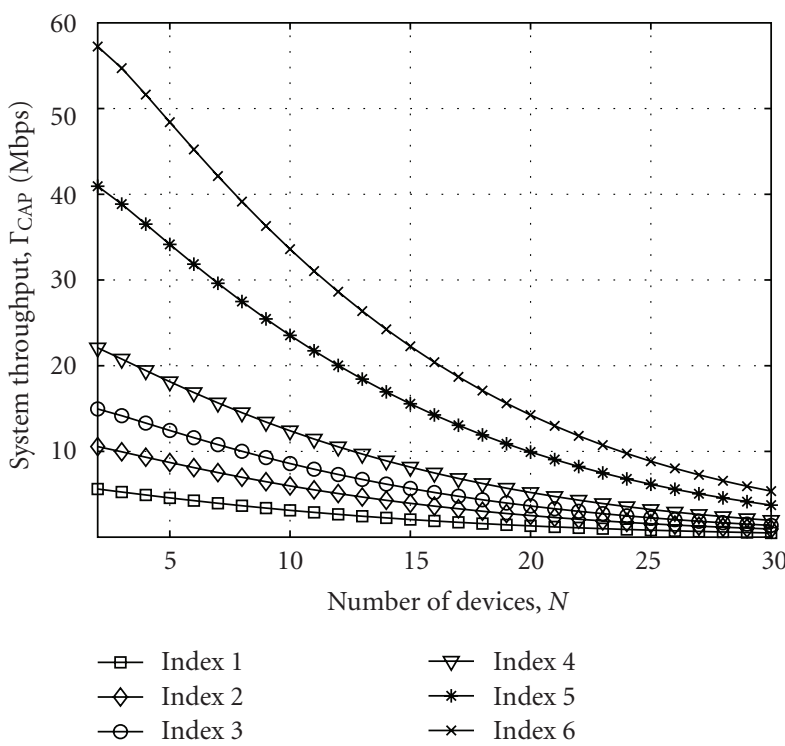

Figure 9: Number of devices versus system throughput in CAP. For Index numbers refer Table 3.

Let $P_{I}, P_{\mathrm{SE}}, P_{C}$ represents the probabilities of idle, success/error, and collision events. In a channel with a total of $N$ devices,

$$
\begin{gathered}
P_{I}=(1-\Lambda)^{N}, \\
P_{\mathrm{SE}}=\left(\begin{array}{c}
N \\
1
\end{array}\right) \Lambda(1-\Lambda)^{(N-1)}, \\
P_{C}=1-P_{I}-P_{\mathrm{SE}} .
\end{gathered}
$$

The normalized system throughput $\Gamma_{\mathrm{CAP}}^{(\mathrm{nor})}(\mathrm{bps} / \mathrm{Hz})$ can be given as

$$
\Gamma_{\mathrm{CAP}}^{(\mathrm{nor})}=\frac{P_{\mathrm{SE}} L_{\mathrm{fr}}(1-\mathrm{FER})}{P_{I} T_{I}+P_{\mathrm{SE}} T_{\mathrm{SE}}+P_{C} T_{C}},
$$

where $L_{\mathrm{fr}}=\Omega_{\mathrm{PHY}} T_{\mathrm{fr}}$ denotes the frame payload size in octets.

Finally, the effective system throughput (bps) can be expressed by

$$
\Gamma_{\mathrm{CAP}}=\Omega_{\mathrm{PHY}} \Gamma_{\mathrm{CAP}}^{(\text {nor })}
$$

where $\Omega_{\mathrm{PHY}}$ is the data rate provided by the PHY layer design.

The specific MAC parameter settings for this analysis is given as follows [14]: for Index 1 to 3 where $f_{\mathrm{nr}}=5 \mathrm{MHz}, \sigma$ is set as $21 \mu \mathrm{s}, \beta$ is set as $64 \mu \mathrm{s}$, and $T_{\text {pre }}$ and $T_{H}$ are 16 and $64 \mu \mathrm{s}$, respectively. As for Index 4 to 6 where $f_{\mathrm{nr}}=20 \mathrm{MHz}$, $\sigma$ is set as $9 \mu \mathrm{s}, \beta$ is set as $16 \mu \mathrm{s}$, and $T_{\text {pre }}$ and $T_{H}$ are 4 and $16 \mu \mathrm{s}$, respectively. Other parameters such as $\alpha$ is equivalent to $\beta+2 \sigma, \delta$ is set as $1 \mu \mathrm{s}$, and $\mathrm{CW}_{\min }$ and $\mathrm{CW}_{\max }$ are set as 16 and 1023. Also note that the ACK frame is 14 octets while the average data frame $L_{\mathrm{fr}}$ is 4096 octets.

Figure 9 presents the relationship between number of devices and achievable system throughput $\Gamma_{\mathrm{CAP}}$. In Figure 9, Index 1 to 3 shows $\Gamma_{\mathrm{CAP}}$ for end-user connectivity, while Index 4 to 6 are particularly designed for backbone connectivity. With low number of devices sharing the channel 
(e.g., $N=2$ ), the efficiency of CAP is at least $75 \%$ for all cases. As $N$ increases, more collision among the users take place and $\Gamma_{\mathrm{CAP}}$ decreases.

In the TDMA scheme, a device conducts communication only in the dedicated time slot allocated by the NC. The TDMA is employed to perform time-sensitive data streaming for better quality of service. Applications that may benefit from this scheme are the online video streaming and peerto-peer video transmission.

Referring to Figure 7, the time slots are scheduled in the CFP, which is the period following the CAP. Devices intending to reserve the GTS in the CFP shall first request for time slot allocation from the NC in the CAP. Figure 8(c) shows the handshakes between the device and the NC for GTS allocation. The device first sends a GTS request frame to the NC, then awaits the ACK frame. If it receives the ACK frame, it will expect the next incoming beacon frame to carry the timing descriptor of the allocated GTS. The device will, therefore, conduct its collision-free streaming in the allocated GTS. The data streaming in GTS takes place as the time of the allocated GTS arrives. As shown in Figure 8(d), the source device will send data to the destination device and receives an ACK frame if successful.

The normalized system throughput $\Gamma_{\mathrm{CFP}}^{(\mathrm{nor})}(\mathrm{bps} / \mathrm{Hz})$ can be given as the ratio between average payload information and the total frame length plus the frame-to-frame interval

$$
\Gamma_{\mathrm{CFP}}^{(\text {nor })}=\frac{E\left[L_{\mathrm{fr}}\right]}{E\left[L_{\mathrm{fr}}\right]+L_{H}+L_{\mathrm{pre}}+\beta} .
$$

Finally, the effective system throughput (bps) can be expressed by:

$$
\Gamma_{\mathrm{CFP}}=\Omega_{\mathrm{PHY}} \Gamma_{\mathrm{CFP}}^{(\text {nor) }} .
$$

The calculation of $\Gamma_{\mathrm{CFP}}$ is more straightforward due to the collision-free transmission. The specific MAC parameter settings for this analysis is given as follows [14]: for Index 1 to 3 where $f_{\mathrm{nr}}=5 \mathrm{MHz}, \beta$ is $64 \mu \mathrm{s}$, and $T_{\text {pre }}$ and $T_{H}$ are 16 and $64 \mu \mathrm{s}$, respectively. As for Index 4 to 6 where $f_{\mathrm{nr}}=20 \mathrm{MHz}, \beta$ is $16 \mu \mathrm{s}, T_{\text {pre }}$ and $T_{H}$ are 4 and $16 \mu \mathrm{s}$, respectively. The average data frame $L_{\mathrm{fr}}$ is 4096 octets.

Employing (21) and the relevant parameters, $\Gamma_{\mathrm{CFP}} \mathrm{calcu}-$ lated for Index 1 through 6 are given as 6.5, 13, 19.6, 27.5, 55 , and $82.5 \mathrm{Mbps}$, respectively. Note that $\Gamma_{\mathrm{CFP}}$ is the average system throughput for the system, where each user may occupy only a certain time slot for respective transmission.

\section{A Combined Analysis}

This section combines all the analysis from the previous sections to outline the entire design flow of the enabling technology for TVWS operation, as described in Section 2 and Figure 1. In Section 3, the use case scenarios are described highlighting both TVWS backbone connectivity and enduser connectivity with applications requiring throughput from $100 \mathrm{kbps}$ up to $2 \mathrm{Mbps}$. The use case scenarios also specify other required parameters such as FER and supported number of users to be 0.08 and 30. In Section 4, the requirements in the regulation such as cognitive radio capability and

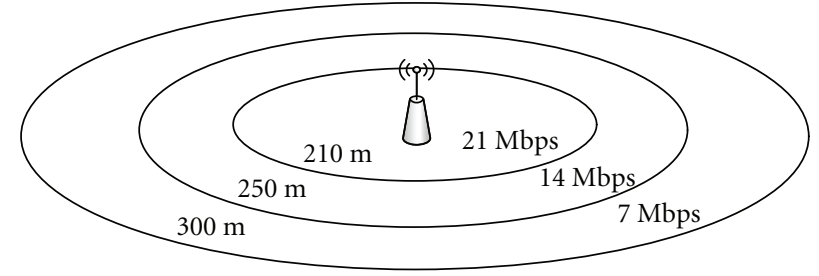

(a) LPD-Index 1 to 3

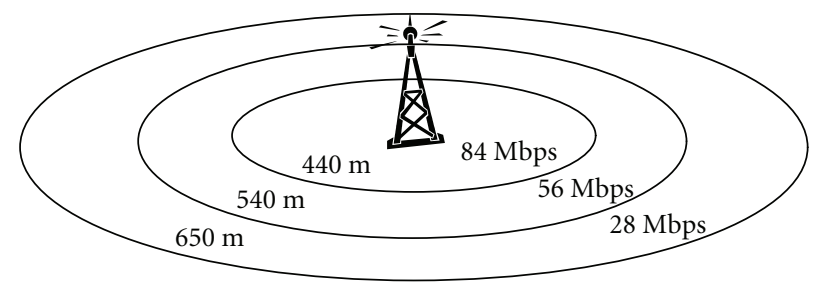

(b) HPD-Index 4 to 6

FIGURE 10: Achievable PHY data rate and operating range for HPD and LPD.

TV channel plan are given. Together, the use case and the regulations define the "perimeter" of requirements for the system design.

The system design consists of the channelization design, the PHY and MAC layer designs. In Section 5.2, the PHY layer design employing OFDM signal is proposed with the output data rates of at least $7 \mathrm{Mbps}$. The minimum achievable operating range is $300 \mathrm{~m}$. A total of six data rate modes (Index 1 to 6) are proposed to support different application demands, as illustrated in Figure 10.

Next, in Section 5.4, the cognitive management engine and other enabling MAC protocols are specified. The cognitive management engine specifies a state transition diagram based on the regulation requirements (i.e., geolocation awareness and spectrum sensing) to control the occupancy of TV channels. Other enabling MAC protocols such as channel access procedures specify the channel access mechanism that facilitates the sharing of the same channel by multiple users. A hybrid CSMA/CA and TDMA channel access method is proposed to support both effective multiple access and quality of service transmissions. In the CSMA/CA method, the minimum and maximum system throughput are 5.6 and 57.2 Mbps, respectively, whereas in the TDMA method, 6.5 and $82.5 \mathrm{Mbps}$, respectively.

Finally, according to Figure 1, the system throughput and operating range are fed back to the use case requirements. With the achievable system throughput, the communication system in TVWS is capable of supporting up to a typical number of 40 users (e.g., assuming simultaneous video streaming) in some $400 \mathrm{~m}$ radius. The supportable number of users that conduct web browsing or other less bandwidth hungry applications may even be higher. Additionally, for applications in need of higher power efficiency, devices with lower complexity and lower power may be employed at the expense of lower number of supported users and shorter range. 


\section{Conclusion}

This paper proposes a cross-layer MAC/PHY layer design for wireless communication system operating in the TVWS, taking into consideration practical issues such as application use cases and governing regulations. The advantages offered by the proposed communication system in supporting the targeted use cases and complying to the regulations are verified. Future works include the specific optimization in each of the design block shown in this paper.

\section{References}

[1] Second Report and Order and Memorandum Opinion and Order: In the Matter of Unlicensed Operation in the TV Broadcast Bands, Federal Communication Commission, Document 08-260, November 2008.

[2] Second Memorandum Opinion and Order: Federal Communication Commission, Document 10-174, September 2010.

[3] Consultation Document: Implementing Geolocation. OFCOM, November 2010.

[4] IEEE Draft Standard 802.11af D1.0, "Wireless LAN MAC and PHY Specications. Amendment 2: TV White Spaces Operation," January 2011.

[5] "MAC and PHY for Operation in TV White Space," ECMA392, ECMA International, 1st edition, December 2009.

[6] IEEE Draft Standard 802.22, "Wireless Regional Area Network MAC and PHY Specications: Policies and procedures for operation in the TV bands," Draft 7.0, December 2010.

[7] Project Authorization Request for SCC41 White Space Radio, September 2010.

[8] J. Gebert and K. Kalliojarvi, "Use cases for operation in white space frequency bands," ETSI RRS WG1.

[9] R. Paine, M. Cummings, and A. Reznik, "White space coexistence use cases," IEEE 802.19.1, doc: IEEE 802.19-09$0026 / \mathrm{r} 4$

[10] D. Lubar, "IEEE SCC41 ad hoc on WS radio: WS use cases," IEEE SCC41 ad hoc on WS radio, doc: scc4i-ws-radio-100010r0.

[11] C. S. Sum et al., "IEEE SCC41 ad hoc on WS radio: White space usage models," IEEE SCC41 ad hoc on WS radio, doc: scc4i-wsradio-10-0005r0.

[12] IEEE Draft Standard 802.15.4g, "Wireless MAC and PHY Specications for Low-Rate WPANs: Physical Layer Specications for LowData Rate Wireless Smart Metering Utility Networks," Draft D2, October 2010.

[13] T. Lukumaa, "MPEG4 and MPEG7," http://citeseerx.ist.psu .edu.

[14] IEEE Standard 802.11 Revision 2007, "Wireless LAN MAC and PHY Specifications," June 2007.

[15] H. Harada and R. Prasad, Simulation and Software Radio for Mobile Communications, Artech House, Boston, Mass, USA, 2002.

[16] V. Erceg et al., "Channel models for fixed wireless applications," doc.: IEEE 802-16-01-0029-04-003c, July 2001.

[17] E. Sofer et al., "WRAN channel modeling," doc.: IEEE 802-2205-0055-07, August 2005.

[18] S. Sampei, Applications of Digital wireless Technologies to Global Wireless Communications Feher, Prentice Hall, New York, NY, USA, 1997.
[19] IEEE Standard 802.15.3, "Wireless MAC and PHY Specications for High-Rate WPANs," September 2003.

[20] IEEE Standard 802.15.4, "Wireless MAC and PHY Specifications for Low-Rate WPANs," September 2006.

[21] T. Li, Q. Ni, D. Malone, D. Leith, Y. Xiao, and T. Turletti, "Aggregation with fragment retransmission for very highspeed WLANs," IEEE/ACM Transactions on Networking, vol. 17, no. 2, pp. 591-604, 2009.

[22] G. Bianchi, "Performance analysis of the IEEE 802.11 distributed coordination function," IEEE Journal on Selected Areas in Communications, vol. 18, no. 3, pp. 535-547, 2000. 

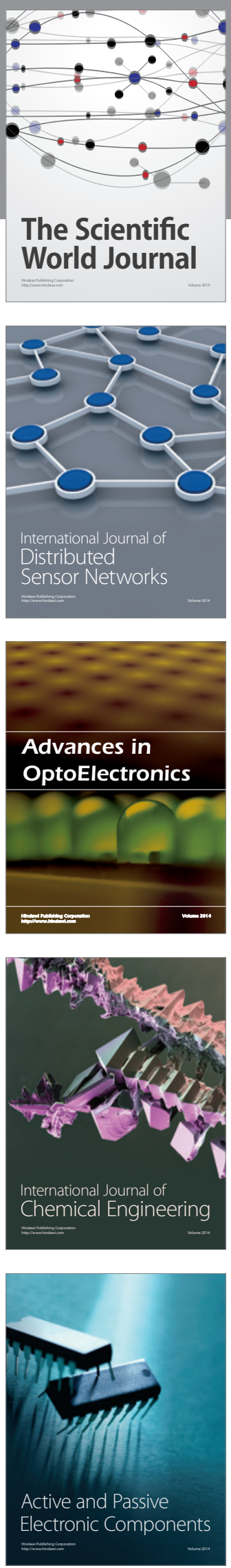
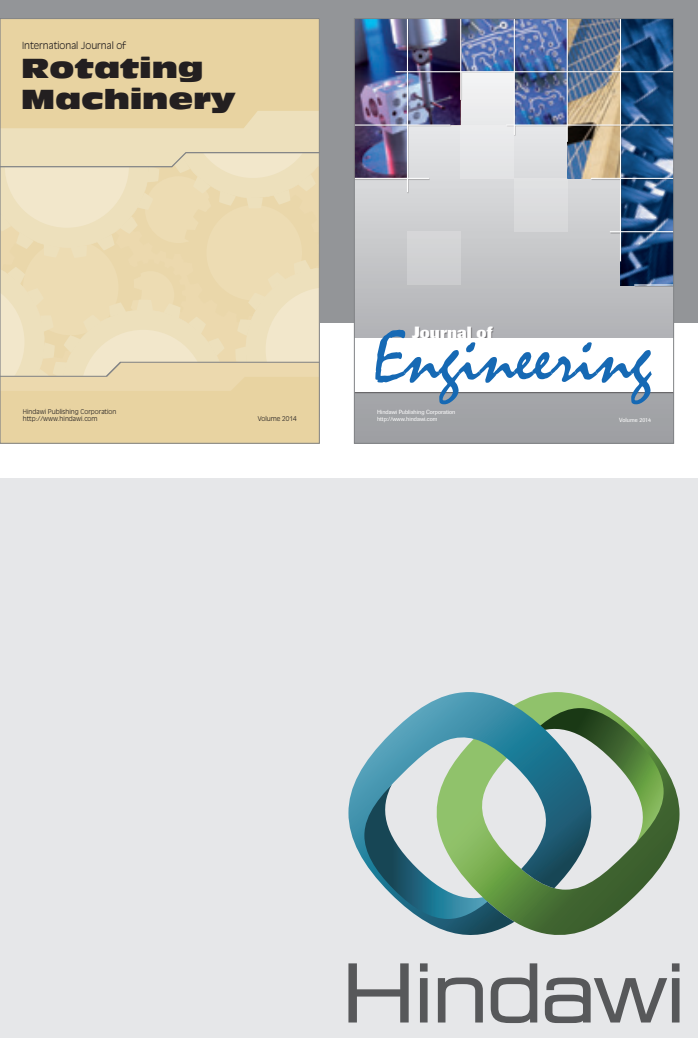

Submit your manuscripts at

http://www.hindawi.com
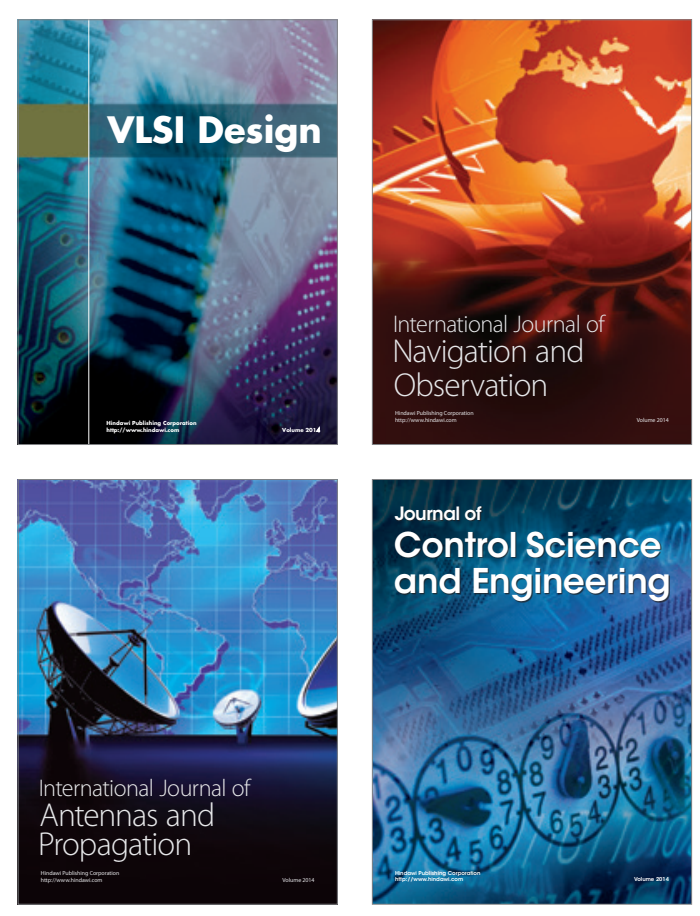
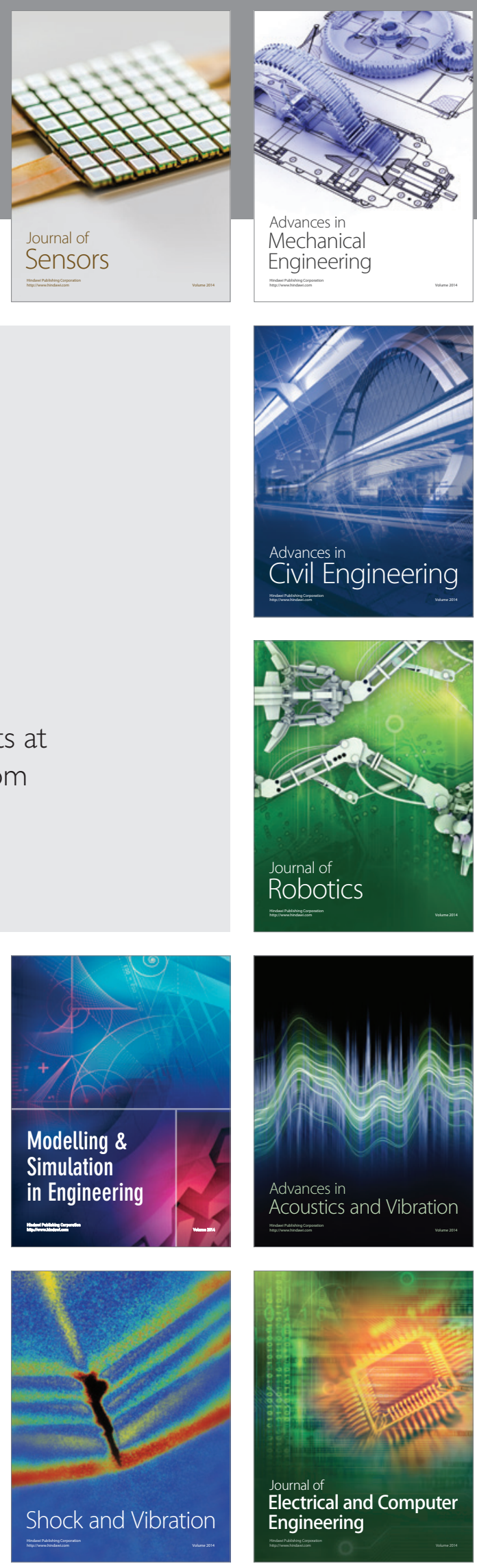